La prise en compte du risque industriel : état et perspectives

Oléagineux, Corps Gras, Lipides. Volume 10, Numéro 1, 5-10, Janvier - Février 2003, La filière, aujourd'hui, demain

Auteur(s) : Fabrice BOSQUE, Xavier PAGES, Institut des Corps Gras - ITERG rue Monge, 33600 Pessac, France.

Résumé : Les auteurs précisent les contraintes législatives liées à l'implantation d'un site industriel en zone urbaine. L'intégration du risque industriel dans la réglementation française est rappelée à travers l'étude de la législation des installations classées pour la protection de l'environnement. Le code de l'environnement et le code de l'urbanisme sont tous deux adaptés pour prévenir l'impact humain et matériel d'un accident industriel. L'explosion de l'usine AZF à Toulouse en septembre 2001 a déclenché une réflexion au niveau national sur la prise en compte du risque en zone urbaine. Nous en présentons ici les principaux axes : propositions de l'inspection générale de l'environnement et de la commission d'enquête parlementaire, le débat national, un projet de loi relatif à la prévention des risques technologiques et naturels et à la réparation des dommages. Cette réflexion devrait aboutir à la mise en place d'une véritable culture du risque intégrant industriels, état, collectivités locales, salariés, et aussi population. L'explosion sur le site industriel de Toulouse en septembre 2001 a suscité une réflexion sur la maîtrise du risque technologique. II nous a semblé intéressant de rappeler la manière dont s'intègre la prise en compte du risque industriel dans la législation française. Nous reviendrons sur l'explosion de Toulouse et sur ses conséquences, puis nous dresserons les perspectives d'évolution de la maîtrise du risque.

\title{
ARTICLE
}

Résumé

Les auteurs précisent les contraintes législatives liées à l'implantation d'un site industriel en zone urbaine. L'intégration du risque industriel dans la réglementation française est rappelée à travers l'étude de la législation des installations classées pour la protection de l'environnement. Le code de l'environnement et le code de l'urbanisme sont tous deux adaptés pour prévenir l'impact humain et matériel d'un accident industriel. L'explosion de l'usine AZF à Toulouse en septembre 2001 a déclenché une réflexion au niveau national sur la prise en compte du risque en zone urbaine. Nous en présentons ici les principaux axes : propositions de l'inspection générale de l'environnement et de la commission d'enquête parlementaire, le débat national, un projet de loi relatif à la prévention des risques technologiques et naturels et à la réparation des dommages. Cette réflexion devrait aboutir à la mise en place d'une véritable culture du risque intégrant industriels, état, collectivités locales, salariés, et aussi population. 
L'explosion sur le site industriel de Toulouse en septembre 2001 a suscité une réflexion sur la maîtrise du risque technologique. II nous a semblé intéressant de rappeler la manière dont s'intègre la prise en compte du risque industriel dans la législation française. Nous reviendrons sur l'explosion de Toulouse et sur ses conséquences, puis nous dresserons les perspectives d'évolution de la maîtrise du risque.

L'intégration du risque industriel dans la législation française

Le risque industriel englobe deux notions distinctes: le risque industriel chronique, regroupant les différentes formes de pollutions susceptibles de présenter un impact sur la santé des populations et sur l'environnement, et le risque industriel accidentel, qui résulte de la présence de produits ou de procédés dangereux susceptibles de provoquer un accident entraînant des conséquences immédiates graves pour le personnel, les riverains, les biens et l'environnement.

La législation européenne traite ces deux types de risque de manière distincte : le risque chronique est pris en compte par la directive IPPC (contrôle et prévention intégrés des pollutions), le risque accidentel est pris en compte par la directive Seveso. En France, l'approche est plus globale : les deux types de risque sont pris en compte dans la législation des installations classées.

La législation des installations classées

Les sites industriels implantés en France sont soumis à la législation des installations classées pour la protection de l'environnement (ICPE). Cette législation, fixée par la loi du 19 juillet 1976, organise le contrôle des activités polluantes et dangereuses, dans un but de maîtrise ou de limitation de l'impact sur l'environnement des installations. II s'agit d'une approche globale qui intègre toutes les nuisances pouvant être occasionnées sur l'environnement : incendies, explosions, bruit, pollution de l'air, de l'eau, pollution résultant des déchets, de la radioactivité, atteintes esthétiques... Cette loi est maintenant codifiée au titre $1^{\mathrm{er}}$ du Livre $\mathrm{V}$ du Code de l'Environnement.

Historiquement, la réglementation des activités industrielles naît en 1810 par un décret impérial daté du 15 octobre, suite à l'explosion d'une poudrerie. Cette législation est aujourd'hui basée sur une nomenclature des installations permettant de les classer selon la notion d'activité (par exemple, la rubrique 2240 concerne l'extraction ou du traitement des huiles végétales, huiles animales, corps gras,...) ou de stockage de substances ou préparations (la rubrique 1136 concerne l'emploi ou le stockage de l'ammoniac). 
Une installation est dite installation classée lorsque l'une de ses activités figure dans la liste de la nomenclature. Suivant les quantités stockées ou la capacité de l'activité, l'installation sera soumise à déclaration ou à autorisation.

Les installations de capacité modeste sont considérées comme présentant des risques peu importants et relèvent donc du régime de la déclaration. Elles doivent respecter des prescriptions techniques générales. Elles peuvent fonctionner dès que le préfet a délivré à l'exploitant un simple récépissé.

Les installations de capacité plus importante sont considérées comme présentant des risques conséquents et sont donc soumises au régime de l'autorisation. Elles doivent respecter des prescriptions techniques spécifiques à l'installation définies dans un arrêté préfectoral d'autorisation. Cet arrêté fixe les conditions d'aménagement et d'exploitation de l'installation, les moyens d'analyse et de mesure pour suivre le fonctionnement de l'exploitation, les méthodes et les moyens d'intervention en cas d'accident, les mesures d'urgence à adopter et les obligations de l'exploitant en matière d'information et d'alerte des personnes concernées. Cette autorisation est délivrée par le préfet sur présentation d'un dossier par l'exploitant et composé notamment d'une étude d'impact et d'une étude de dangers. Ces deux études exposent les mesures de prévention et d'évaluation des incidences en situation normale et en situation accidentelle. Ce dossier fait l'objet d'une étude approfondie de la part des services préfectoraux avant la délivrance de l'autorisation d'exploiter. Le risque chronique et le risque accidentel sont donc bien considérés à ce stade.

Les prescriptions de fonctionnement sont fixées avant le démarrage de l'installation. En cours d'exploitation, les prescriptions peuvent être modifiées par voie d'arrêté complémentaire.

Certaines installations, du fait des risques importants qui leur sont liées, sont susceptibles de donner lieu à des servitudes d'utilité publique. Elles sont visées par les textes transposant en droit national les directives européennes dites Seveso I et Seveso II. La première (directive du Conseil du 24 juin 1982) est abrogée depuis le 3 février 1999 suite à l'entrée en vigueur des dispositions de la seconde (du 9 décembre 1996) concernant la maîtrise des dangers liés aux accidents majeurs impliquant des substances dangereuses. La seconde directive dispose d'un champ d'application plus étendu et renforce les dispositions relatives à la prévention. Elle a été transposée en droit français par le décret du 20 mars 2000 et l'arrêté du 10 mai 2000 (une circulaire datée du même jour précise l'application de cet arrêté). Les installations dites "Seveso" sont considérées comme particulièrement dangereuses et doivent impérativement disposer d'un plan d'opération interne (POI) et d'un plan particulier d'intervention (PPI) qui précisent la prise en compte des accidents potentiels par les exploitants et les pouvoirs publics et la mise en place d'un dispositif global de prévention des risques. 
La réglementation des installations classées concerne 500000 installations soumises à déclaration et environ 65000 installations soumises à autorisation. Le total des établissements visés par la directive Seveso II s'élève à environ 1250.

L'application de la législation des installations classées est donc de la responsabilité directe de l'Etat (les préfets par délégation), contrairement à d'autres pays où différentes entités juridiques peuvent intervenir (état, région, département, commune).

La directive Seveso I a vu le jour suite à l'accident de Seveso le 10 juillet 1976. La législation des installations classées est née et a évolué au fil des accidents industriels... II nous semble donc nécessaire de dresser le profil des possibilités d'évolution législative suite à l'accident sur le site industriel de Toulouse.

Installations classées et urbanisme

La maîtrise de l'urbanisation à proximité des sites industriels constitue un axe de prévention des risques. En effet, la réduction du nombre des personnes exposées et la conception des bâtiments sont des facteurs essentiels. La relation population/site industriel a donc toujours été au centre de la réglementation concernant les installations classées, et a motivé sa création. La question se pose alors en terme d'interaction Droit de l'Urbanisme/Droit de l'Environnement.

Le Code de l'urbanisme permet au préfet de limiter la construction autour des sites industriels en délimitant un périmètre spécifique indépendamment d'un plan local d'urbanisme (PLU, anciennement appelé POS, plan d'occupation des sols). Ce Code prévoyait des dispositions limitant I'urbanisation autour des installations présentant des risques technologiques. La loi SRU, loi de décembre 2000 relative à la solidarité et au renouvellement urbain instituant les PLU, a fait disparaître cette mention. La notion de risque technologique reste néanmoins présente dans le Code qui précise que les plans locaux d'urbanisme déterminent les conditions permettant d'assurer notamment la prévention des risques technologiques, des pollutions et des nuisances de toute nature. Les documents graphiques du PLU font apparaître les secteurs où les nécessités du fonctionnement ou de risques technologiques justifient que soient interdites ou soumises à des conditions spéciales les constructions et installations.

Un PLU peut donc être dénoncé quand il y a manifestement une erreur d'appréciation des risques encourus.

Mais la disposition du PLU n'est pas le seul moyen de prévenir une trop grande proximité entre population et industrie à risque. Un permis de construire peut toujours être refusé s'il y a risque estimé, sans pour autant qu'il y ait un périmètre de protection de défini, comme le précise la partie 
réglementaire du Code de l'Urbanisme: "Le permis de construire peut être refusé ou n'être accordé que sous réserve de l'observation de prescriptions spéciales si les constructions, par leur situation ou leurs dimensions, sont de nature à porter atteinte à la salubrité ou à la sécurité publique. Il en est de même si les constructions projetées, par leur implantation à proximité d'autres installations, leurs caractéristiques ou leur situation, sont de nature à porter atteinte à la salubrité ou à la sécurité publique."

Si les autorités municipales n'ont pas estimées suffisamment le risque industriel, l'autorité préfectorale, par institution d'un PIG (projet d'intérêt général), peut limiter l'urbanisation à proximité d'un site dans un souci de prévention des risques. Le PIG s'impose alors au PLU et l'état doit veiller à sa prise en compte dans le document d'urbanisme local.

Ces mesures ne prévoit cependant pas des possibilités d'indemnisation, sauf s'il résulte de ces servitudes une atteinte à des droits acquis ou une modification à l'état antérieur des lieux déterminant un dommage direct, matériel et certain.

Rappelons que "le permis de construire peut être refusé ou n'être accordé que sous réserve de l'observation de prescriptions spéciales si les constructions, par leur situation ou leurs dimensions, sont de nature à porter atteinte à la salubrité ou à la sécurité publique. Il en est de même si les constructions projetées, par leur implantation à proximité d'autres installations, leurs caractéristiques ou leur situation, sont de nature à porter atteinte à la salubrité ou à la sécurité publique."

En enfin, le Code de l'Urbanisme, dans sa partie législative, précise que "le permis de construire ne peut être accordé que si les constructions projetées sont conformes aux dispositions législatives et réglementaires concernant l'implantation des constructions, leur destination, leur nature,...". Le Code de l'Urbanisme renvoie donc au Code de l'Environnement, qui indique que la demande de permis de construire est établie en même temps que la demande d'autorisation d'exploiter. Une circulaire précise les conditions d'obtention du permis de construire dans le cas des installations classées.

Le droit de l'environnement gère donc également la cohabitation installations classées/population. Des servitudes d'utilités publiques sont possibles dans le cas des installations dites "Seveso" et doivent être annexées au PLU. Ces servitudes sont applicables uniquement dans le cas des installations à implanter sur un site nouveau et ne peuvent contraindre "à la démolition ou à l'abandon de constructions existantes édifiées en conformité avec les dispositions législatives et réglementaires en vigueur avant l'institution desdites servitudes". Contrairement aux dispositions du Code de l'Urbanisme, ces servitudes peuvent donner droit à indemnisation versée par l'exploitant.

Dans un cas plus général, le Code de l'Environnement précise que la délivrance de l'autorisation préfectorale pour les installations classées peut être subordonnée notamment à leur éloignement 
des habitations, immeubles habituellement occupés par des tiers, établissements recevant du public ou des zones destinées à l'habitation par des documents d'urbanisme opposables aux tiers.

Les moyens législatifs et réglementaires existent. Le Code de l'Environnement et le Code de l'urbanisme sont tous deux adaptés pour prévenir l'impact humain et matériel d'un accident. L'arsenal législatif gère de manière efficace la cohabitation industrie à risque/population dans le cas des implantations nouvelles, mais l'urbanisation près des sites existants reste un problème à ce jour sans solution. Le problème vient également d'une application trop laxiste des textes. En effet, de nombreux PLU permettent une urbanisation à proximité d'installations classées pouvant être jugées à risque.

La catastrophe de Toulouse

Le 21 septembre 2001, sur le site de l'usine Grande Paroisse à Toulouse, est survenue une explosion dans un stockage de 300 à 400 tonnes de "nitrate d'ammonium déclassé". Cette explosion cause la mort de 30 personnes (dont 22 dans l'usine) et en blesse 2500 autres. Elle intervient dans un contexte mondial particulier, quelques jours seulement après les attentats du 11 septembre aux Etats-Unis. Les causes ne sont pas connue à ce jour, mais il semble s'agir d'un accident.

A proximité immédiate du site de l'usine de Grande Paroisse se trouvent deux autres sites : la société SNPE (ex Société Nationale des Poudres et Explosifs) et Tolochimie, ainsi que des établissements accueillant du public (hôpital psychiatrique, lycée).

Les effets de la déflagration sont observables sur plusieurs kilomètres autour du site, et notamment dans le centre de la ville de Toulouse. Très vite se pose alors la question de la prise en compte réelle des conséquences d'un accident sur un site industriel lors de la délivrance de l'autorisation d'exploiter.

L'usine Grande Paroisse emploie 470 personnes sur un terrain de 70 ha, pour produire des engrais et divers produits chimiques (production totale estimée à $5000 \mathrm{t} / \mathrm{j}$ ).

Elle est soumise à la législation des ICPE sous le régime de l'autorisation. L'usine était préalablement réglementée par un arrêté préfectoral daté du 12 février 1996, complété par un arrêté du 9 septembre 1998 (suite à une importante fuite d'ammoniac) et un arrêté du 30 mai 2000 concernant spécifiquement les aéroréfrigérants (risque de légionellose).

En 1999, la société a déposé une demande d'extension des capacités de production (ammoniac, urée, acide nitrique). Le nouvel arrêté, daté du 18 octobre 2000, autorise cette extension et 
comporte un ensemble complet des prescriptions techniques (42 pages) applicables à l'établissement (ce qui évite de se rapporter à des arrêtés des prescriptions générales ou arrêtés types).

Selon cet arrêté, le société est autorisée à exploiter les installations listées dans un tableau reprenant 32 rubriques de la nomenclature des installations classées. Pour 7 de ces rubriques listées, le régime imposé est celui de l'autorisation donnant lieu à des servitudes d'utilité publique (classement "AS" dans la nomenclature). II s'agit donc d'un établissement "Seveso II - seuil haut". Le risque d'explosion du nitrate d'ammonium n'a pas été pris en compte dans l'étude de dangers réalisée par l'exploitant.

Les conséquences matérielles de l'accident sont les suivantes : déformation des structures (dégâts aux construction, bris de vitres) qui conduit à estimer que la puissance de cette explosion est comparable à celle de 20 à 40 tonnes de TNT (explosif de référence dans les calculs pyrotechniques).

L'explosion a provoqué la formation d'un cratère d'une quarantaine de mètres de diamètre et de $7 \mathrm{~m}$ de profondeur par rapport au sol naturel. La partie nord du site de l'usine a été dévastée par l'explosion ; cela a entraîné la destruction de certains réservoirs de solutions de nitrate d'ammonium et une pollution de la Garonne, ainsi que des fuites d'acide nitrique. Le réservoir de solution chaude de nitrate d'ammonium à $95 \%$, situé à proximité, a été endommagé mais sans provoquer de fuite. L'explosion ne s'est pas propagée aux autres stockages de nitrate d'ammonium de cette zone, car ceux-ci ont été protégés par des bâtiments ayant résisté à l'onde de choc. On peut considérer que l'effet domino a été limité.

La SNPE a subit des dégâts notables et déplore un mort parmi le personnel, mais aucune des installations techniques n'a été endommagée (il n'y a eu aucune fuite). Les procédures de sécurité ont pu être appliquées de manière satisfaisante. Le site de Tolochimie, légèrement plus éloigné, n'a pas subit non plus de dommages spécifiques.

Notons que la direction de l'usine, très affaiblie (choc psychologique dû notamment aux nombreuses victimes, destruction des locaux et des documents), s'est trouvée confrontée à une très grande difficulté de gestion de la crise, notamment en ce qui concerne l'évacuation des produits stockés sur le site. Face à la confusion des rôles respectifs des pouvoirs publics et l'exploitant, les aides provenant des autres sites du groupe n'ont pu être possibles qu'après intervention du Ministère chargé de l'Environnement, l'exploitant devant prendre les mesures nécessaires pour assurer la sécurité du site, y compris après un accident.

Les faits relatés ici à partir des documents officiels (rapports de I'Inspection Générale de I'Environnement et de la Commission d'enquête parlementaire) mettent en évidence la difficulté à envisager dans les études préalables l'ensemble des scénarii d'accidents susceptibles de survenir sur un site industriel et la difficulté pour l'exploitant à gérer une crise lors d'une catastrophe anéantissant les moyens et matériels dont il dispose. 
L'évolution des dispositifs relatifs a la réduction du risque industriel

La catastrophe survenue sur le site industriel de Toulouse a généré une réflexion sur le risque industriel en général et la mise en place d'un projet législatif. Cette réflexion concerne principalement les sites industriels considérés comme les plus "risqués", les sites classés "Seveso", mais elle devrait à plus ou moins long terme se traduire par une évolution des prescriptions relatives à l'ensemble des installations classées.

De cette réflexion, souvent menée dans un climat passionnel, justifié par l'ampleur de la catastrophe, nous avons extrait les éléments susceptibles d'avoir une incidence directe sur l'activité industrielle.

Les propositions de l'inspection générale de l'environnement

Le 24 septembre 2001, Yves COCHET, alors Ministre de l'aménagement du territoire et de l'environnement, a diligenté une mission de I'Inspection générale de l'environnement relative à l'explosion survenue sur le site industriel de Toulouse.

Ce rapport, qui a été remis au Ministre le 24 octobre 2001, dresse un bilan précis des conditions administratives de fonctionnement de l'usine, des contrôles effectués par l'inspection des installations classées, des conditions d'urbanisation autour du site ainsi que des connaissances sur le nitrate d'ammonium. Le texte du rapport est disponible sur le site web du Ministère :

http ://www.environnement.gouv.fr/infoprat/Publications/publi-ige.htm.

Le rapport émet surtout plusieurs recommandations de portée générale pour améliorer la politique de maîtrise des risques industriels dans les domaines suivants: réglementation du nitrate d'ammonium, effectifs de l'inspection des installations classées, examen critique des études de dangers, gestion de l'urbanisation au voisinage des industries à risque, amélioration de l'information et de la participation du public, test des plans d'urgence.

Concernant l'action de l'inspection des ICPE, le rapport demande le doublement des effectifs de l'inspection des installations classées pour améliorer le contrôle des établissements prioritaires (définis par circulaire) mais aussi pour contrôler les autres établissements. Les auteurs du rapports souhaite mettre en place un deuxième niveau de priorité permettant de porter une attention plus soutenue à des installations qui présentent des risques d'accidents ou des risques pour la santé publique moins importants: installations Seveso "seuil bas", silos. Ils estiment que toutes les installations soumises à autorisation ou à déclaration doivent faire l'objet d'un effort de contrôle. Ils souhaitent également le renforcement des liens entre l'inspection des ICPE et l'inspection du travail. 
Le rapport demande l'amélioration de la qualité des études de dangers : fixation de règles relatives aux accidents et aux agressions extérieures à prendre en compte, aux modalités des analyses de dangers et aux critères définissant les effets sur les personnes. Il est proposé une évaluation de la population habitant dans les diverses zones de risques permettant d'orienter les décisions à prendre. Les études de dangers doivent être expertisées de manière indépendante.

Concernant l'urbanisation à proximité des sites à risques, les auteurs considèrent que la poursuite de certaines activités ne sera possible à long terme que si l'on peut, par des mesures techniques, réduire les risques à un niveau tolérable, compte tenu de l'urbanisation actuelle. Dans les autres cas, il est envisagé la fermeture de l'usine avec un délai de quelques années pour faciliter la transition en veillant à ce que les échéances annoncées soit effectivement tenues.

Concernant la maîtrise de l'urbanisation, les auteurs demandent à ce que les servitudes d'utilité publique ne s'appliquent pas uniquement aux installations à implanter sur un site nouveaux : ils souhaitent élargir le champ d'application de cette disposition, non seulement aux nouvelles installations (sur un site existant), mais également aux installations anciennes. Les dispositions proposées ne permettraient pas de contraindre à démolir des constructions existantes, mais il s'agirait de prévoir pour les zones les plus exposées un droit d'expropriation ou de préemption au profit de l'exploitant, à ses frais, de manière à réduire progressivement l'habitat dans ces zones.

Concernant l'information du public, celle-ci doit être améliorée, par la définition des modalités d'information sur les risques de l'ensemble d'un établissement lors des enquêtes relatives à des extensions (on constate dans la jurisprudence que cette information est souvent limitée pour des raisons de confidentialité). Le rapport demande à ce que le contenu minimal du résumé non technique soit précisé et qu'il comporte au moins une carte faisant apparaître les zones à risque : risque létal à $1 \%$ et risque d'effets irréversibles avec pour chaque zone la population concernée.

Concernant les plans particuliers d'intervention (PPI), les auteurs souhaitent que ceux-ci contiennent des prescriptions précises sur leur révision régulière, l'information, les exercices, la préparation des établissement exposés à l'accident éventuel. Les auteurs demandent la mise en place d'un dispositif de contrôle de l'existence de consignes de sécurité particulières.

Notons que ce rapport a très largement influencé les premières mesures prises suite à l'accident (effectif des inspections), mais également le projet législatif (relatif à la maîtrise des risques technologiques).

Les propositions de la commission d'enquête parlementaire

Constituée le 24 octobre 2001, la Commission d'enquête a achevé ses travaux le 29 janvier 2002 par l'adoption d'un rapport, et s'est attachée à formuler 90 propositions permettant de lutter plus efficacement contre le risque d'accident industriel et de mieux protéger les personnes en cas 
d'accident. Nous reprenons ici les principaux éléments de ce rapport concernant l'exploitation d'un site industriel, le texte complet étant disponible sur le site web de l'Assemblée Nationale :

http ://www.assemblee-nationale.fr/rap-enq/r3559/r3559-023.asp.

Le sentiment de la Commission aux termes de ses travaux est que la conscience des risques, d'une entreprise à l'autre, d'un secteur à un autre, semble très inégale, comme semblent l'être également les moyens consacrés à la politique de sûreté. La Commission a été très favorablement impressionnée par l'attention portée aux questions de sûreté dans de nombreux établissements qu'elle a visités. Elle a toutefois constaté que certains secteurs, en particulier le nucléaire et dans une moindre mesure la pétrochimie, paraissaient en avance sur d'autres en matière de culture de sûreté : plus il y a de dangers potentiels comme dans le nucléaire, plus les règles de sûreté sont respectées, alors que lorsque les risques ne sont pas perçus, comme cela peut être le cas dans les stockages en vrac, il y a une sorte de banalisation et de sous-estimation du risque.

La Commission a également été frappée par l'abondance des règles en vigueur pour prévenir les accidents industriels. La directive Seveso, paraît, pour l'essentiel, satisfaisante. La législation française sur les installations classées qui la transpose et la complète le cas échéant, n'a pas à être bouleversée. II n'est pas jugé nécessaire de faire de la sédimentation juridique, en ajoutant de nouvelles strates réglementaires, sans chercher au préalable à appliquer les textes existants.

La Commission a constaté "un certain décalage entre le discours des organisations professionnelles et de certaines entreprises présentant systématiquement la sécurité comme une priorité absolue et la réalité de pratiques encore trop souvent caractérisées par une pression croissante des impératifs de rentabilité et par la marginalisation des salariés".

La Commission estime toutefois que le champ couvert par le projet de loi est trop restreint, car il concerne presque exclusivement les questions liées à l'urbanisme, à l'information du public et aux modalités d'association des représentants des salariés à la prévention des risques.

Les propositions de la Commission concernent six grands domaines :

la réduction du risque à la source,

le rôle du facteur humain et notamment des salariés dans la prévention des accidents,

la mise en Ïuvre d'une plus grande transparence et d'une expertise pluraliste des risques,

les questions d'urbanisme,

l'indemnisation des victimes de catastrophes industrielles,

l'adaptation des procédures judiciaires. 
Concernant spécifiquement les possibilités de réduction à la source, la commission propose, à défaut de pouvoir modifier les procédés de fabrication, une réduction de la taille des installations, permettant ainsi de réduire les quantités de produits dangereux présentes sur un site. Le fractionnement et l'enfouissement des stocks constitue également une solution, ce qui ne doit pas pour autant contribuer à l'augmentation des risques liés au transport.

Parmi les 90 propositions formulées par la commission, nous avons sélectionné celles nous paraissant concerner davantage les exploitants.

Les études de dangers :

préciser, dans les études de dangers, la liste des personnes impliquées dans leur réalisation et décrire les méthodes utilisées,

actualiser l'étude de dangers en cas de modification de l'organisation du travail.

Les seuils de classements de la nomenclature :

réviser à la baisse les seuils de quantités de produits de la nomenclature des installations classées, corriger les effets de seuil dans la réglementation Seveso pour en garantir l'efficacité

Le management de la sécurité : soutenir les travaux de normalisation des systèmes de management de la sûreté industrielle.

concernant le retour d'expérience :

instituer une obligation de transmission de tout événement touchant la sûreté d'une installation classée Seveso,

durcir fortement les sanctions prévues en cas de non-déclaration des événements touchant à la sûreté des installations Seveso.

L'inspection des installations: doubler, à missions constantes, les effectifs de l'inspection des installations classées.

La représentation des salariés (près de vingt propositions traitent directement du rôle des CHSCT) :

étendre la négociation collective aux contraintes spécifiques qui résultent des activités présentant de graves dangers pour la sécurité,informer et réunir obligatoirement le CHSCT dès le lancement d'une étude de dangers ou d'une révision d'étude de dangers, ainsi que pour la préparation des plans 
d'organisation interne et à l'occasion de la mise en place des services permanents de secours,

permettre au CHSCT de se faire assister par un expert lors de la réalisation des études de dangers.

L'intervention en cas d'accident: imposer la réalisation d'au moins un exercice annuel de mise en Ïuvre du POI en modifiant le décret du 21 septembre 1977.

\section{L'urbanisation :}

aider les exploitants à financer certaines opérations permettant une réduction sensible des périmètres de dangers,

étendre, quand un enjeu d'urbanisme ou de développement économique le justifie, la possibilité d'exonération de la taxe professionnelle à toutes les installations présentant des risques faisant l'objet de modifications substantielles permettant de réduire ceux-ci,

créer un fonds de prévention des risques industriels, financé par l'Etat et les exploitants, exerçant un droit de préemption dans les zones à risque et aidant les investissements de sûreté,

dès que les périmètres de dangers seront fiables, donner à tout propriétaire d'un bien menacé le droit d'imposer l'acquisition de celui-ci par le fonds de prévention des risques industriels.

Le dispositif répressif :

qualifier de délit les infractions aux prescriptions préfectorales ou ministérielles en matière de fonctionnement des installations soumises à autorisation,

harmoniser les pratiques des DRIRE concernant leur intervention auprès des parquets pour le suivi des procès-verbaux d'infractions,

permettre la publicité d'un jugement de condamnation par diffusion télévisée,

renforcer de manière significative, au sein de l'Ecole de la magistrature l'enseignement, initial et professionnel, portant sur le droit de l'environnement et des installations classées,

créer dans toutes les juridictions une section du parquet et une chambre, spécialisées dans les infractions relatives à l'environnement et aux installations classées.

La commission considère que les risques en France sont très mal évalués et qu'une politique foncière ambitieuse doit être mis en place en ce qui concerne l'urbanisation. La priorité absolue doit toutefois être la réduction des risques à la source pour réduire les périmètres de dangers. Les pouvoirs publics doivent donc veiller au renforcement des mesures de sécurité, avec la multiplication des mesures de précaution dans le cadre d'une stratégie de défense en profondeur contre les risques industriels. 
L'intérêt de l'industrie est sans aucun doute de se conformer aux prescriptions des pouvoirs publics et à la demande croissante de l'opinion. En tout état de cause, les pouvoirs publics doivent adopter une démarche offensive, impliquant des obligations précises.

Le débat national

Une réflexion sur les risques industriels a été engagée, à l'initiative du Ministère chargé de I'Environnement, sous forme de tables rondes régionales et nationale, réunissant industriels, population, organisations syndicales, collectivités, scientifiques et services de l'état. Les débats régionaux ont réunis, entre novembre et décembre 2001, environ 7000 personnes, dont $23 \%$ d'industriels.

L'objectif du débat a été de définir une stratégie d'action permettant d'élever globalement le niveau de sécurité industrielle en France et dégager des axes de réformes à mener en matière de prévention. Cette stratégie peut se traduire par des mesures de prévention des risques industriels au niveau organisationnel, réglementaire et législatif. Elle porte à la fois sur :

les impératifs de sécurité du personnel et de la population de proximité,

la préoccupation du développement urbain,

les conditions du maintien et du développement des industries à risque.

Les débats ont été menés autour de trois axes principaux :

production, sécurité et surveillance des sites industriels,

sites à risque et développement urbain,

information et participation du public.

Parmi les différentes actions proposées lors des tables rondes régionales, nous avons sélectionné celles ayant été reprises lors du débat national qui concernent plus particulièrement la gestion d'un site industriel et qui risquent d'orienter à plus ou moins long terme l'évolution de la réglementation.

Propositions générales :

mieux identifier les risques à la source,

prendre plus nettement en considération les impératifs de sécurité, non seulement lors de la conception des installations mais aussi dans le cadre de leur maintenance et de leur évolution,

créer une échelle de risque pour l'évaluation des accidents industriels concernant l'ensemble de installations classées, 
mener une réflexion sur la nécessité des produits "dangereux" et les processus de fabrication "à risques",

mettre en place un label environnemental pour distinguer les produits dont la fabrication présente des risques de ceux dont la fabrication est considérée comme "sûre",

fragmenter les sites de stockage et reconsidérer les conditions de stockage,

développer les technologies aux moindres risques,

développer la mise en place des systèmes de management de la sécurité et les audits de sécurité interne.

Gestion du personnel :

assurer une meilleure sensibilisation et formation au risque pour les salariés, sous-traitants et intérimaires,

limiter le recours à la sous-traitance et au travail intérimaire,

étendre les prérogatives des CHSCT sans pour autant désengager la responsabilité de l'exploitant en matière de sécurité,

organiser des visites croisées des salariés entre différents sites (partage d'expériences, regard extérieur).

Etudes de dangers :

faire des études de dangers un outil majeur de prévention,

exiger des tierces expertises par des cabinets indépendants,

introduire une obligation de renouvellement des études dans le temps pour toutes les installations.

Retour d'expérience : mieux recenser les accidents, mais aussi les "presque accidents" et créer des banques de données facilement accessibles.

Responsabilité et sanction :

renforcer la responsabilité civile et pénale de l'industriel,

impliquer davantage les assureurs dans la gestion de la sécurité industrielle,

rendre les sanctions plus dissuasives. 
Contrôle des DRIRE :

accroître les moyens des DRIRE en effectifs et en compétence technique,

développer les visites inopinées,

renforcer l'expertise indépendante sur l'évaluation des risques et des impacts,

mettre en place une certification des organismes pour les tierces expertises.

Sites industriels et développement urbain :

privilégier la réduction du risque à la source plutôt que la délocalisation,

contraindre les entreprises à acquérir les terrains inclus dans les périmètres de danger

simplifier et rendre plus applicables les consignes du POI (Plan d'Opération Interne) et du PPI (Plan Particulier d'Intervention),

mieux articuler et établir une continuité totale entre POI et PPI,

pratiquer un exercice annuel de mise en Ïuvre du PPI par zone industrielle,

intégrer les retours d'expérience des exercice du POI et du PPI.

Information du public :

instaurer un dialogue permanent entre industriels, pouvoirs publics et population (hors contexte de crise),

encourager un financement partagé de l'information,

donner un rôle de médiation aux élus entre industriels et population,

ne pas négliger le rôle des organisations syndicales et des salariés dans leur rôle spontané de médiation entre industriels et population,

informer le citoyen afin qu'il puisse devenir un acteur de sa propre sécurité,

étendre les règles d'information du public imposées aux entreprises classées "Seveso" à toutes les installations jugées "dangereuses",

développer des relais pour l'information,

généraliser les réunions publiques avec débat contradictoire,

réaliser régulièrement des journées "Portes ouvertes",

développer une véritable culture du risque, 
améliorer la clarté et la compréhension de l'information, vulgariser l'information technique du risque,

vulgariser les études de dangers sans pour autant occulter l'information pertinente,

engager les industriels à un travail régulier de communication avec la population,

réactualiser fréquemment les brochures d'information,

communiquer sur "l'utilité" des produits fabriqués, afin de mieux faire accepter le risque éventuel.

Ces débats ont donné lieu à un rapport d'ensemble de la part du coordinateur, Philippe Essig, rapport remis au Premier Ministre fin janvier 2002. Les limites de la réglementation sont dénoncées : la directive "Seveso II" est mise en cause dans sa pertinence et ses possibilités d'application dans les zones urbaines. Philippe Essig propose la création une Délégation à la Sécurité Industrielle (DSI) visant au développement de la recherche fondamentale en matière de cindynique (discipline qui évalue et tente de prévenir les dangers induits par une activité économique), à la mise en place de référentiels pour les études de dangers (avec une approche probabiliste), et au développement du management de la sécurité. L'auteur appelle au développement d'audits externes pour les études de dangers qui serviraient de bases aux analyses des CLIRT (proposés par le projet de loi). La Délégation à la sécurité industrielle devra également mener une réflexion sur le transport des marchandises dangereuses. Enfin, le rapport insiste sur la nécessité de participation active des citoyens dans la gestion du risque.

le rapport complet est disponible sur Internet à l'adresse suivante :

http ://www.environnement.gouv.fr/dossiers/risques/risques-industriels/20020206-rapportessig.htm

Le projet de loi

n premier projet de loi, concernant le renforcement de la maîtrise des risques technologiques pour les installations susceptibles de donner lieu à des servitudes d'utilité publique ("Seveso seuil haut"), avait été déposé au Sénat en février 2002, lors de la précédente législature. Ce premier projet comportait trois volets :

le renforcement des instruments de maîtrise de l'urbanisation autour des sites à risques,

la concertation entre les acteurs concernés localement par le risque industriel,

l'amélioration de la prévention et de la gestion des risques par une meilleure implication des représentants du personnel et des intervenants extérieurs.

Suite aux élections législatives de juin 2002, ce premier projet a été abandonné par le gouvernement Raffarin, au profit d'un projet incluant également les risques naturels, et en particulier les inondations. 
Le nouveau projet de loi, relatif à la prévention des risques technologiques et naturels et à la réparation des dommages, est largement inspiré du premier, mais aussi des propositions de la commission d'enquête parlementaire (voir plus haut).

Concernant sa partie "risque industriel", ce dernier projet est orienté autour de quatre axes :

l'information du public: création de commissions locales d'information et de concertation (CLIC) autour de chaque site à risque,

l'urbanisation: mise en place de moyens pour éviter l'aggravation des situations existantes et remédier aux situations déjà graves; le principe général sera l'indemnisation par l'industriel de la servitude créée par toute augmentation du risque de son fait; des plans de prévention des risques technologiques (PPRT) seront mis en place autour des sites à risque ("Seveso seuil haut"); cette nouvelle loi créé un "droit de délaissement": les riverains des sites Seveso existants auront désormais la possibilité de demander à la commune de racheter leur maison à un prix fixé par l'administration des domaines, suivant des zones définies par les PPRT; les frais occasionnés seront partagés entre les collectivités locales, l'état et les industriels.

participation des salariés et des sous-traitants à la gestion du risque : élargissement du rôle du comité d'hygiène, de sécurité, et des conditions de travail (CHSCT), meilleur encadrement du recours à la sous-traitance (liste de postes réservés aux salariés de l'entreprise en contrat à durée indéterminée, définition de mesures de précaution et de sécurité, formation d'accueil, représentation des entreprises extérieures au CHSCT...),

l'indemnisation: ouverture d'un droit à réparation immédiate, sans franchise, et dans des délais encadrés par la loi.

Les exploitants des installations "Seveso seuil haut" auront également obligation de réaliser, en complément de l'étude de danger, une évaluation quantitative des dommages aux tiers que pourraient provoquer les installations dangereuses en cas d'accident. D'autre part, le rapport annuel présenté à l'assemblée générale des sociétés exploitant des installations à risque devra contenir des informations sur le niveau de risque présenté par l'entreprise, ainsi que les dispositions prises pour en assurer la prévention et organiser, le cas échéant, l'indemnisation des victimes.

Ce projet a été voté par le Sénat le 6 février 2003. L'intégralité du texte est disponible sur Internet à l'adresse suivante :

http ://www.environnement.gouv.fr/actua/

com2003/janvier/3-loi-risques-index.htm

Vers une culture du risque?

Cette prise de conscience des outils existants, dont l'augmentation des effectifs des DRIRE est une des manifestations, et cette volonté de les améliorer peut amener à développer une véritable culture du risque. Le renforcement de l'information du public est aussi un moyen efficace d'améliorer l'intégration des sites industriels dans un environnement urbain, et d'apaiser des craintes parfois 
irrationnelles, afin de pouvoir passer d'un risque ressenti par la population comme subi à un risque accepté.

Si l'aspect technique reste essentiel, notamment par une véritable réflexion sur les possibilités de réduction à la source, une prévention efficace ne peut s'envisager que par l'intégration d'un management du risque: ne plus considérer l'étude de dangers comme une simple démarche administrative mais comme une base de gestion, utiliser les outils organisationnels proposés par les textes réglementaires (par exemple, le SGS, système de gestion de la sécurité transposé de la directive Seveso II dans l'arrêté du 10 mai 2000), développer l'application de référentiels de management de la sécurité et du management intégré qualité/sécurité/environnement, et enfin gérer le risque dans sa globalité. Rappelons à ce titre que la directive Seveso II intègre la notion d'établissement et non plus d'installation, et prends en compte également "l'effet domino" (nécessité d'examiner les conséquences d'un accident sur les installations voisines). En effet, le risque industriel n'est pas localisé uniquement sur le site industriel. Toutes les infrastructures de transport présentent également un risque industriel. Les procédures de réduction des volumes de stockage de produits, parfois réalisés dans un réel souci de réduction du risque, mais le plus souvent motivés pour s'affranchir de certaines contraintes réglementaires, ne doit pas générer une augmentation des risques liés au transport. 